\title{
QAM to Circular Isomorphic Constellations
}

\author{
Farbod Kayhan \\ Interdisciplinary Centre for Security, Reliability and Trust (SnT), \\ University of Luxembourg \\ (email: farbod.kayhan@uni.lu).
}

\begin{abstract}
Employing high order constellations is inevitable in order to achieve higher spectral efficiency in satellite communication systems. In DVB-S2X standard, constellations with up to 256 points have been included. However, optimizing such high order constellations is a difficult task. In this paper we propose a very simple constellation design based on the radial map from QAM constellations to non-uniform APSK constellations. Our method provides a systematic way to generate a family of optimized high order constellations. We show that gains larger than $0.5 \mathrm{~dB}$ can be obtained with respect to the DVBS2X constellations with 256 points. Even though the proposed constellations are essentially sub-optimal (in terms of maximizing the mutual information), they provide a very competitive test benchmark for the performance of high order constellations. Moreover, the proposed construction potentially allows for an efficient one-dimensional soft detection by an inverse mapping to the QAM. Finally, further optimization techniques are discussed.
\end{abstract}

Index Terms-Non-uniform APSK constellations, High order modulation, soft demapping, DVB-S2X standard

\section{INTRODUCTION}

In view of the growing demand for spectral efficiency in satellite communications, high order modulation design has received a considerable attention in the past few years. To cater to this demand, modulation and coding (MODCOD) configurations employing constellations of orders 64, 128 and 256 were adopted in the new digital video broadcasting standard (DVB-S2X) [1]. Such high order constellations are also under considerations by Consultative Committee for Space Data Systems (CCSDS) for future standards. Given the fact that the traffic demand for satellite broadband is expected to grow six-fold by 2020 [2], and the continuous need for higher data rates in satellite communications, even larger constellations may be needed in near future.

A distinctive property of satellite communication systems, is the non-linear characteristics of high power amplifier (HPA) on-board of the satellite. Hence, the conventional additive white Gaussian noise (AWGN) channel with average power limitation is no longer an accurate model and nonlinear characteristics have to be taken into account. This usually leads to assume a peak power limited signaling due to the HPA operating near the saturation limits. Under the peak power constraint, the capacity achieving distribution is proved to be discrete in amplitude (having finite number of mass points) with a uniformly distributed phase [3]. Even though the optimal distribution for a finite set is not known in general case, several previous studies indicate that amplitude and phase-shift keying (APSK) modulations perform very close to the capacity
[4]-[6]. Recently, it has been shown that asymptotically, APSK constellations can also achieve the Gaussian capacity [7].

Optimizing the APSK modulation has been studied by several authors in the literature. The number of points on each circle, the radii and phases of each concentric circle of APSK constellations need to be optimized in order to achieve near capacity performances [6]. Even though for constellations with up to 64 points, the number of possibilities is rather limited and therefore the optimization problem can be still handled, for larger constellations the problem becomes complex and the proposed algorithms in the literature usually fail to provide a good solution.

Another problem which arises regarding the optimization of APSK constellation is the bit to symbol mapping. This problem becomes particularly important in the pragmatic systems, where no iteration between the detector and the decoder is allowed [8]. On top of that, when the pragmatic approach is considered, the APSK constellations are usually not the best choices [9]. Following this observation, several algorithms have been proposed in the literature for constellation design without imposing any particular structure (see for example [10]). Recently, a simulated annealing algorithm [11] has been implemented to maximize the average mutual information (AMI) [9] or the pragmatic average mutual information (PAMI) (also known as the BICM capacity). AMI and PAMI, if they can be computed with a good approximation, provide a reliable metric to assess the performance of a given constellation. When the objective function is PAMI, one needs to jointly optimize the constellation and the corresponding labelling in order to assecure a good performance over a pragmatic system.

Simulated annealing becomes inefficient for constellations larger than 64 . However, by limiting the optimization to only a quadrant (by assuming symmetry) and initializing the optimization algorithm by a good constellation (such as QAM) one can optimize constellations up to 256 points for a variety of channel models and constraints [12]. For larger constellations, however, the simulated annealing becomes extremely slow and may converge to a local maximum which is only slightly better than the initializing constellation.

In this paper we propose a simple construction for nonuniform APSK constellations, based on the radial map between squares and circles. The obtained constellations are then the image of $M$-QAM. We refer to these constellations as QAM to circular isomorphic (QCI) constellations. Our main goal is to introduce a family of constellations which can serve as a competitive benchmark for future high order constellation 
design under the peak power constraint. As we will show, the proposed constellations (without any further optimization) may provide even more than $1.5 \mathrm{~dB}$ gain with respect to the constellations employed in DVB-S2X. These results should also highlight the importance of having such a testing benchmark in future standardization.

Beside serving as a benchmark, the QCI constellations actually provide the state-of-art performance for peak power limited channels to the best knowledge of the author. We also discuss briefly several ways to improve and built upon the results presented in this paper. An optimization strategy is discussed which allows to optimize constellations with up to $2^{24}$ points. Moreover, being the image of the QAM, the QCI constellations potentially allow for a very efficient onedimensional (1D) soft-detection by inverse mapping to the QAM constellation.

The rest of this paper is organized as follows. In Section II we describe the notations and the objective functions upon which the constellations are compared. We also describe the QCI construction in details. In Section III we compare the AMI and PAMI of QCI constellation with those of DVB-S2X. We discuss some of the ongoing and future research directions in Section IV. Finally, we conclude the paper in Section V.

\section{QAM TO CIRCULAR ISOMORPHIC CONSTELLATIONS}

A constellation $\chi$ is simply a finite subset of the $k$ dimensional Euclidean space, i.e., $\chi \subset \mathbb{R}^{k}$. In this paper, we are mainly interested in constellations with $M=2^{m}$ elements and $k=2$, even though the results can be easily generalized to the higher dimensions and non-binary constellations. The elements of $\chi$ are referred to as constellation points or transmitted symbols. The symbols are associated to the bits at the input of the modulator through a one-to-one labelling $\mu: \chi \rightarrow\{0,1\}^{m}$. For any given symbol $\mathbf{x}$, we denote by $\mu^{i}(\mathbf{x})$ the value of the $i^{t h}$ bit of the label associated to it.

It is well-known that squares and circles are isomorphic, i.e., there exists a map between them which is continuous, one-to-one and onto with continuous inverse. We are interested in one of the simplest existing maps, i.e. the radial mapping or concentric mapping (see Fig. 1). This isomorphism maps

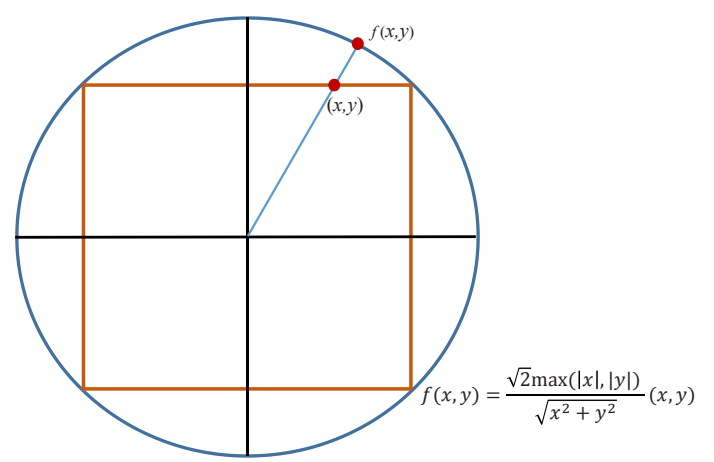

Fig. 1. Square to circle isomorphic radial map.

the concentric squares into the concentric circles. The same map can be used to project the unit square onto a Disc. In more details, let $S=\{(x, y)|| x|\leq 1| y \mid, \leq 1\}$ and $C=$ $\left\{(x, y) \mid x^{2}+y^{2} \leq 2\right\}$ denote the unit square and the disc with radios $\sqrt{2}$, respectively. Then the radial map $f: S \rightarrow C$ can be written analytically as below:

$$
f(x, y)=\left\{\begin{array}{cc}
\frac{\sqrt{2} \max (|x|,|y|)}{\sqrt{x^{2}+y^{2}}}(x, y) & \text { if }(x, y) \neq(0,0) \\
(0,0) & \text { if }(x, y)=(0,0)
\end{array}\right.
$$

In Fig. 2 we plot the 256-QAM and the image of it under the radial isomorphism. We denote the inverse mapping by $f^{-1}=1 / f$ for $(x, y) \neq(0,0)$. It is important to notice

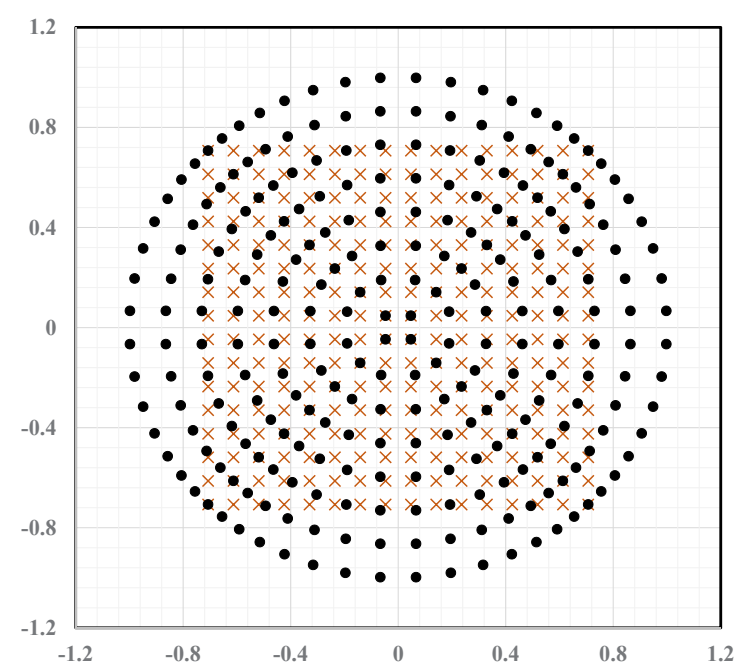

Fig. 2. The 256-QCI constellation generated by mapping 256-QAM to the non-uniform 256-APSK.

that the binary Gray labelling of QAM constellation will be preserved under $f$, and the resulting QCI constellation has also a Gray labelling. For each $M$-QAM constellation, a unique $M$ QCI constellation can be constructed. For even values of $m$, the $M$-QCI constellation has $2^{m / 2-1}$ equidistant concentric rings. The number of points on each ring form an arithmetic progression starting from 4 and with common difference 8 . For odd values of $m$, the resulting QCI constellation has essentially the same structure, except for the last few circles (outer rings).

\section{A. Performance metric}

For the AWGN channel, the received signal can be written as $\mathbf{y}=\mathbf{x}+\mathbf{n}$, where $\mathbf{n}$ has a Gaussian distribution. AMI and PAMI have been shown to be reliable metrics for measuring the constellation performance for a given channel [5]. We denote, respectively, by $I(\chi)$ and $I_{p}(\chi, \mu)$ the AMI and PAMI of a given constellation $\chi$ and mapping $\mu$ :

$$
I(\chi)=\frac{1}{M} \sum_{\mathbf{x} \in \chi} E_{\mathbf{n}}\left\{\log \frac{P(\mathbf{y} \mid \mathbf{x})}{P(\mathbf{y})}\right\}
$$




$$
\begin{aligned}
I_{p}(\chi, \mu) & =\sum_{i=1}^{m} I\left(\mu^{i}(\mathbf{x}) ; \mathbf{y}\right), \\
& =\frac{1}{M} \sum_{i=1}^{m} \sum_{\mathbf{x} \in \chi} E_{\mathbf{n}}\left\{\log \frac{P\left(\mathbf{y} \mid \mu^{i}(\mathbf{x})\right)}{P(\mathbf{y})}\right\}
\end{aligned}
$$

where $I(.,$.$) is the mutual information function and E_{\mathbf{n}}$ is the average with respect to the Gaussian noise.

\section{COMPARISON With DVB-S2X}

The DVB-S2X standard implements various constellations with 256 points ( 3 of which are APSK), but to the best of the author's knowledge, no justification about the constellation design has been provided [1]. In this section we compare the AMI and PAMI of the 256-QCI with the DVB-S2X constellations.

As mentioned, we are interested in the peak-power limited design and therefore in what follows we always fix the maximum power of a constellation to be 1 , i.e., $|\mathbf{x}|^{2} \leq 1$ for all $\mathbf{x} \in \chi$. In such cases, instead of the signal to noise ratio, the peak power to noise ratio (PSNR) should be considered as a measure for comparing two constellations. Notice that PSNR is the ratio between the peak power of the constellation and the noise power spectral density

$$
\mathrm{PSNR} \triangleq \frac{1}{N_{0}} \geq \mathrm{SNR} \triangleq \frac{1}{M} \sum_{\mathbf{x} \in \chi} \frac{|\mathbf{x}|^{2}}{N_{0}} .
$$

Assuming a memoryless ideal non-linearity model (soft limiter) for the HPA and ignoring the effect of filters, the PSNR coincides with $P_{\text {sat }} / N_{0}$ :

$$
\mathrm{SNR}=\frac{E_{s}}{N_{0}}=\frac{P_{\mathrm{sat}}}{N_{0}} \cdot \frac{E_{s}}{P_{\mathrm{sat}}}=\mathrm{PSNR}-O B O[\mathrm{~dB}] .
$$

Six MODCODs in DVB-S2X use constellations with 256 points. However, two of the MODCODs use the same constellation, and therefore, only five different constellations with 256 points exist in DVB-S2X standard [1]. These five constellations can be distinguished by the rate of the codes that they are coupled with. For example, we denote the constellation used with the LDPC code of rate $135 / 180$ by "DVBS2X 135/180". Three of the constellations are APSK with 8 concentric circles each having 32 points. The "DVBS2X 20/30" and "DVBS2X 22/30" have somehow irregular shapes and are probably obtained by initializing an optimization algorithm with an APSK structure mentioned above.

In Fig. 3 we compare the AMI of the 256-QCI and all five constellations of DVB-S2X. At 1 bit lower than the saturation point, QCI shows a gain of $0.7 \mathrm{~dB}$ with respect to the best constellation in DVB-S2X. Targeting lower spectral efficiency, the gain reduces to $0.5 \mathrm{~dB}$. We also plot the peak-powerlimited capacity [3] in the same figure. Notice the loss of QCI constellation with respect to the capacity is around 0.1 $\mathrm{dB}$ for a rather wide range of PSNRs.

As for the PAMI values, we plot the curves in Fig. 4. The gains are a little bit smaller with respect to the best
DVB-S2X constellation. In Fig. 5 we replot both AMI and PAMI results for the 256-QCI, the best and the worst DVBS2X constellations for the most relevant range of spectral efficiencies.

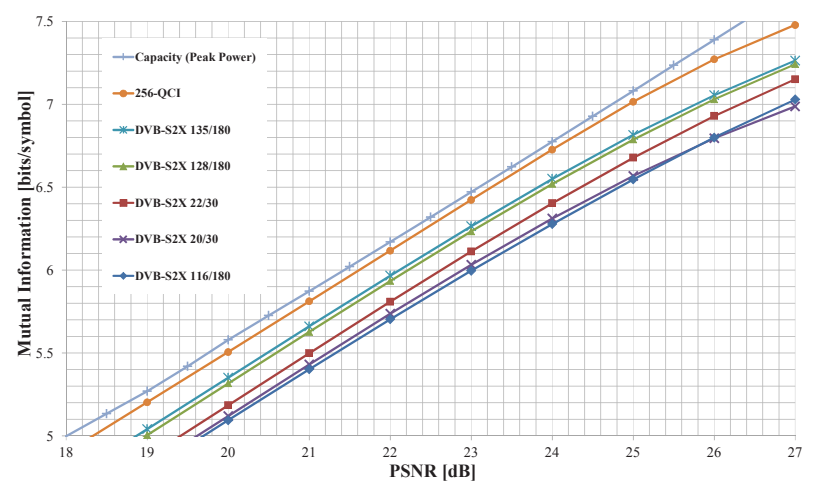

Fig. 3. AMI comparison between 256-QCI and all DVB-S2X constellations.

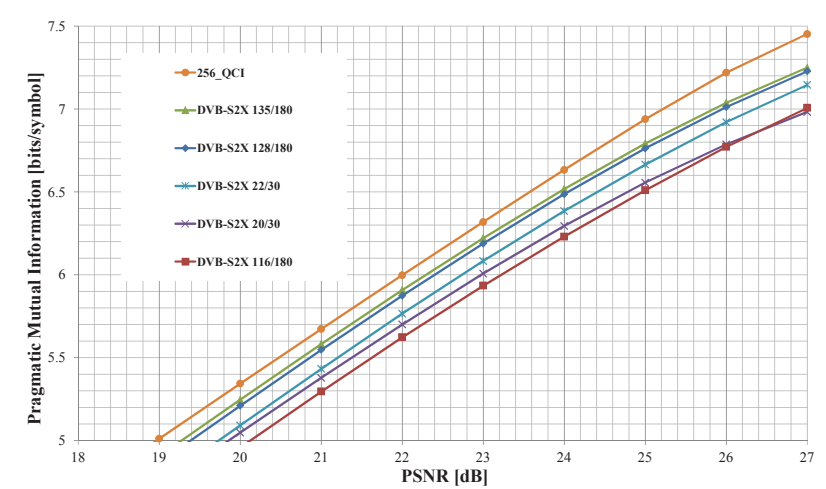

Fig. 4. PAMI comparison between 256-QCI and all DVB-S2X constellations.

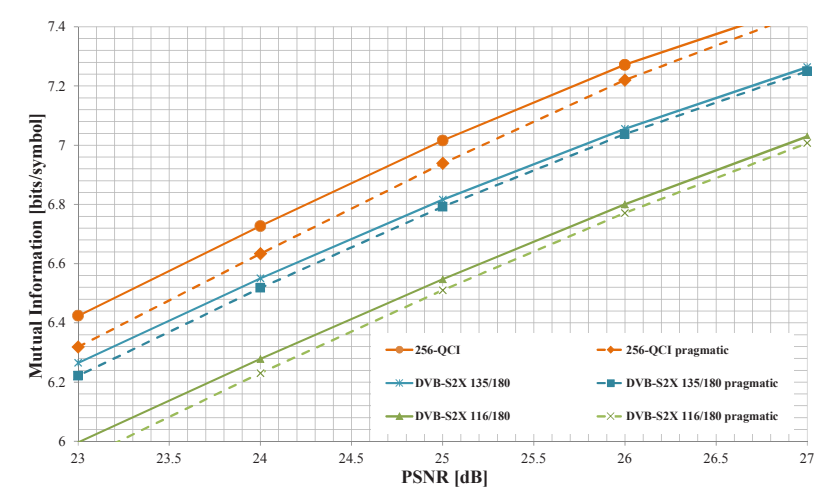

Fig. 5. AMI and PAMI comparison with DVB-S2X constellations.

\section{Discussion AND Future Studies}

\section{A. $1 D$ optimization of QCI constellations}

As mentioned before, the AMI of the QCI constellations achieve within $0.1 \mathrm{~dB}$ from the peak-power limited capacity. However, the PAMI loss is larger (around $0.4 \mathrm{~dB}$ ). It is rather 
easy to find constellations with higher PAMI. In particular, initializing the simulated annealing algorithm by QCI and carefully choosing the cooling parameters, normally results in a constellation with slightly higher PAMI, in compromise, the inverse mapping to the QAM constellation will not be anymore valid, a characteristic which can be important in order to reduce the detection complexity. On the other hand, over the AWGN channel with average-power constraint, the QCI constellations suffer the same asymptotic loss as QAM constellations (around $1.5 \mathrm{~dB}$ ).

Given the QAM structure and the characteristics of the function $f$, the nearby rings of a QCI constellation have the same distance from each other. One can however optimize the radii of the rings. This allows to further optimize the QCI constellations for a wide range of channel models and parameters, while preserving the 1D-detection property.

The radii optimization for $\mathrm{M}-\mathrm{QCI}$ can be interpreted as a 1D constellation design with $2^{(m / 2)-1}$ points (when $m$ is even). For example, as for 256-QCI, one needs to find the placement of only 8 points over the positive $x$-axis which maximizes the AMI (PAMI) of resulting 256-QCI constellation with non-uniform distribution of the radii (see Fig. 6). Notice that the points over the outer circles tend to be more uniformly distributed in Fig. 6 for the optimized constellations. Simulated annealing can be used for this 1D optimization problem. This algorithm provides very competitive solutions for $1 \mathrm{D}$ constellation designs with up to 64 points ([12], [11]). Therefore, constellations as big as $2^{24}$-QCI may be optimized using this strategy.

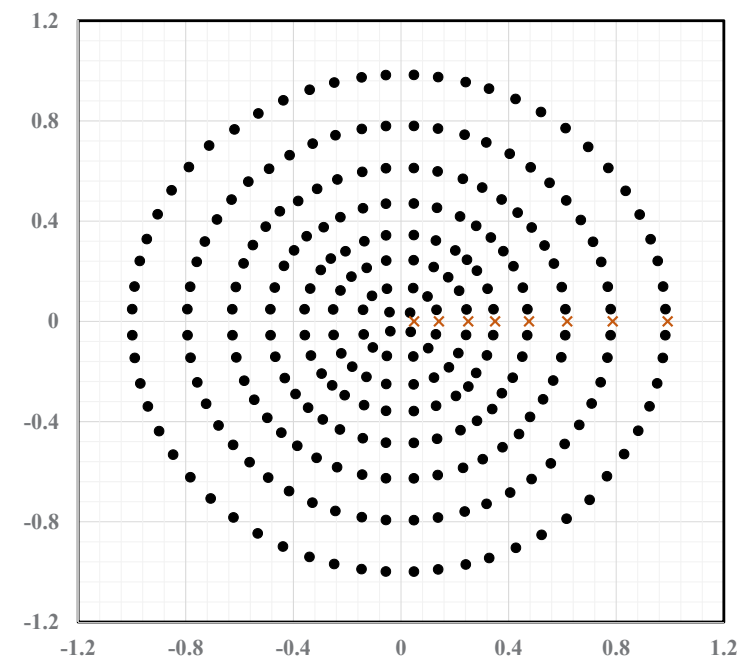

Fig. 6. The 256-QCI constellation generated using a non uniform 256-QAM constellation.

Optimizing the radii of the QCI constellations may also provide us with a good family of constellations for AWGN under the average-power constraint. Some research in this direction is ongoing.

\section{B. Low complexity $1 D$ soft detection}

One of the main drawbacks of employing large constellations is the receiver's (detector and decoder) high computational complexity. The log-likelihood ratios (LLR) need to be calculated at the detector and sent to the soft-decoder. As for the complexity of the decoder, some recent studies indicate that hard decoding of all bits except two or three bits in each dimension will not cause significant losses in the performance [13].

On the other hand, the complexity of the detector grows exponentially as a function of $m$ (number of bits associated to each symbol). Therefore, having a low complexity soft detector is crucial when high order modulations are employed. Being the Cartesian product of two 1D constellations, the complexity of the detector grows only polynomially with respect to $m$ for QAM constellations. For QCI constellations, a straightforward strategy for low complexity detection is first to compute $f^{-1}(y)$, and then calculating LLRs assuming a QAM constellation. A block diagram for such a system is shown in Fig. 7.

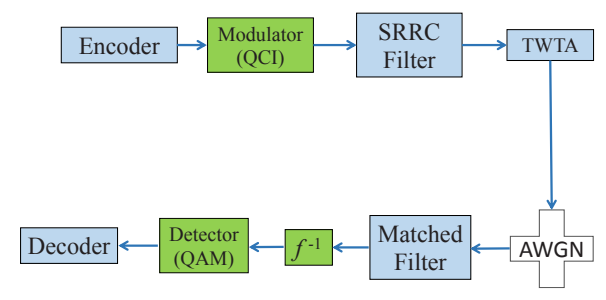

Fig. 7. Block diagram for low complexity detection of QCI constellations.

More elaborated techniques may be proposed for 1D soft detection of the QCI constellations. For example, techniques similar to those in [14] for computing the 1D soft demapping for rotated QAM constellations may be used with appropriate modifications. Finally, notice that the radial map is not the only (up to scaling) isomorphism between squares and circles. Other isomorphisms may be considered that may map the QAM into a uniform APSK constellation or any other desired shapes. For all such constructions an inverse mapping will provide a $1 \mathrm{D}$ detection, however the loss with respect to the optimal maximum likelihood detector may be large depending on the isomorphism. Moreover, it may be difficult to express such isomorphims in closed from analytically. Anyway, it is an interesting problem to find a non-trivial isomporphism family which causes a small loss in 1D detection.

\section{End-to-end simulation results for DVB-S2X chain using QCI Constellations}

Previous studies show that the AMI and PAMI values, calculated under the peak power constrain, predict with a very good accuracy the simulation results over DVB-S2X channel model [9]. However, the end-to-end chain simulations are still needed to ensure that the mapping chosen for the constellation matches the optimized LDPC code through the bit interleaved 
pattern (see in [1]) and no error floor exists. Research in this direction is ongoing.

\section{Conclusions}

In this paper we propose a simple construction for a family of non-uniform APSK constellations by the radial isomorphism between squares and circles. The average mutual information values indicate gains larger than $0.5 \mathrm{~dB}$ with respect to the DVB-S2X constellations with 256 points. This family also provides a competitive test benchmark for future high order modulation design over the satellite channel. We also propose a strategy to further optimize QCI constellations. The construction inherently allows for a simple and fast 1D soft detection by an inverse mapping to QAM constellations. As for the ongoing research, we are simulating the DVB-S2X chain employing our proposed constellation to confirm the gains which are observed in terms of mutual information. Another interesting direction is to provide analytical expressions for the symbol error rate of QCI constellations.

\section{ACKNOWLEDGMENT}

The author would like to thank Alberto Mengali, Bhavani Shankar and Bjorn Ottersten for fruitful and inspiring discussions.

\section{REFERENCES}

[1] ETSI 302-307-2, "Digital Video Broadcasting (DVB) Part II: DVBS2Extensions (DVB-S2X)," 2014.

[2] "Broadband Access via Integrated Terrestrial and Satellite Systems (BATS). D4.1: Satellite Network Mission Requirements," Technical Report, European Project, Avanti PLLC, 2012.
[3] S. Shamai (Shitz) and I. Bar-David, "The capacity of average and peak-power-limited quadrature Gaussian channels," IEEE Transaction on Information Theory, vol. 41, pp. 1061-1071, July 1995.

[4] S. Ikeda, K. Hayashi, and T. Tanaka, "Capacity and modulations with peak power constraint," arXiv:1005.3889 [cs.IT], May 2011.

[5] F. Kayhan and G. Montorsi, "Joint signal-labeling optimization under peak power constraint," Int. J. of Satellite Communications and Networking, DOI: 10.1002/sat.1016, 2012.

[6] R. De Gaudenzi, A. Guillen i Fabrigas and A. Martinez, "Performance analysis of turbo-coded APSK modulations over nonlinear satellite channels," IEEE Trans. Wireless Communications, vol. 5, pp. 23962407, 2006.

[7] H. Meric, "Approaching the Gaussian Channel Capacity With APSK Constellations" in IEEE Communications Letters, vol. 19, no. 7, pp. 1125-1128, July 2015 .

[8] G. Caire, G. Taricco, and E. Biglieri, "Bit-interleaved coded modulation," IEEE Transaction on Information Theory, vol. 44, pp. 927-946, May 1998.

[9] F. Kayhan and G. Montorsi, "Constellation design for transmission over non-linear satellite channels," in IEEE Glob. Telecommun. Conf. (GLOBECOM), Annaheim, USA, Dec. 2012.

[10] A. J. Kearsley, "Global and local optimization algorithms for optimal signal set design," J. Res. Nat. Inst. Stand. Technol., vol. 106, pp. 441$454,2001$.

[11] C. D. G. S. Kirkpatrick and M. P. Vecchi, "Optimization by simulated annealing," Science, vol. 220, pp. 671-680, May 1983.

[12] F. Kayhan and G. Montorsi, "Constellation design for memoryless phase noise channels",IEEE Transactions on Wireless Communications, vol.13, no.5, pp.2874,2883, May 2014.

[13] G. Montorsi and F. Kayhan, "Analog Digital Belief Propagation and its Application to Multi-Stage Decoding Systems," 2015 IEEE International Black Sea Conference on Communications and Networking (BlackSeaCom), pp.82-86, May 2015.

[14] K. Kim, N. Basutkar, K. Bae, P. Xue and H. Yang, "One-Dimensional Soft-Demapping Algorithms for Rotated QAM and Software Implementation on DSP,' IEEE Transactions on Signal Processing, vol. 61, no. 15, pp. 3918-3930, Aug.1, 2013. 